\title{
Exocrine gland cysts in tracheal and bronchial walls
}

\author{
Krishtafovich $\mathrm{AA}^{1}$ and Ariel $\mathrm{BM}^{2 *}$ \\ ${ }^{1}$ City of St Petersburg State Bureau for Pathology, Russia \\ ${ }^{2}$ St Petersburg Federal Research Institute of Phthisiology and Pulmonology, Healthcare Ministry of Russian Federation, Russia
}

\begin{abstract}
Background: To investigate the occurrence of exocrine gland cysts in the tracheal and bronchial walls of patients with chronic lung diseases.

Objectives: 68 patients with lung carcinomas, pulmonary tuberculosis and chronic non-neoplastic lung diseases were examined. The airway mucosa was visualized using an original powder spraying device (Patent RF № 2053801) and $5 \mathrm{~g}$ of aerosolized bioinert $\leq 1 \mu \mathrm{m}$ tantalum particles per assay.

Results: X-ray images taken immediately, 24 and 48 hours after the particle spraying showed the sedimentation of tantalum particles on the mucosal surface and in the excretory ducts of the exocrine glands. Multi-layer bands up to $3 \mathrm{~mm}$ in thickness were found immediately after spraying; duct dilatation and retentional cysts 3-10 mm in size were observed in the exocrine glands. The number of cysts ranged from 1 to 6 per patient. "Risk zones", their arrangement and dimensions were identified as well as the particle concentration in these zones.
\end{abstract}

Conclusion: The changes above likely represent progressive stages of cyst development in excretory ducts of the tracheal and bronchial exocrine glands.

\section{Introduction}

Some parts of trachea and bronchi are particularly predisposed to the development of inflammatory diseases and tumors. It is caused primarily by an uneven deposition of aerogenic pollutant particles, tobacco smoke and many other harmful for the epithelium sediments on the mucosa of these organs. In 95 per cent of cases, exocrine glands in the wall of the respiratory tract serve as anatomical base for the development of adenomas, cancer and other diseases [1]. It is known that there are millions mouths of tiny excretory ducts of the exocrine glands on the surface of tracheal and bronchial mucosa where billions of different particles settle out, damaging the epithelium [2].

To study the patterns of sedimentation of pollutants and other aerogenic particles on the surface of the tracheal and bronchial mucosa, various instruments and methods were used both in vivo and in vitro, animal experiments and mathematical models including [3]. However, neither bronchofibroscopy, nor conventional radiation techniques, nor other research methods allowed to impartially visualize the deposition of particles on the mucosa and reveal "risk zones". We have not found any reports in the literature on the visualization of "risk zones" and cystlike structures in tracheal and bronchial walls. Hence there is no reliable information about "risk zones", their formation mechanisms, precise location, size and boundaries, as well as about deposited particles concentration on the surface of the epithelium and their impact on the exocrine glands. This makes it difficult to detect cysts and conduct differential diagnostics of the airway mucosa injuries characteristic of a particular pathology.

We proceeded from the obvious assumption that according to the laws of physics and aerodynamics, particles that penetrate the respiratory tract from the environment share one common trait: identical deposition trajectory in complex ducts [4]. Therefore, using radiopaque tantalum microparticles in inhalation aerosol as markers, it is possible to obtain on the X-ray films the image of general relief of the upper and lower airways mucosa that would simulate the precipitation of various small particles on the epithelium surface. Moreover, this $\mathrm{X}$-ray model would allow not only studying the mechanisms of particle sedimentation on the mucosal surface, but also detecting "risk zones" there.

Thus, the aim and purposes of the study are to work out a method for X-ray visualization of "risk zones" and open cysts of exocrine glands and to describe the roentgenomorphological features they exhibit when tantalum microparticles are introduced into the lumen of the trachea and bronchi by an inhalation device.

\section{Methods}

68 patients aged 19 to 67 with various kinds of lung pathology (chronic non-specific lung disease, cancer, tuberculosis) were examined. In 12 cases $(17.6 \%)$, open cysts of exocrine glands were accidentally found on the X-ray films of the trachea and major bronchi. The bronchofibroscopy performed in 27 (39.7\%) patients revealed mouths of excretory ducts of the exocrine glands on the lower walls of the main and lobar bronchi. To visualize the mucosal relief with cysts, radiopaque bioinert microparticles of tantalum with a maximal size of $1 \mu \mathrm{m}$ in the amount of $5 \mathrm{~g}$ were used. The aerosol introduction into the trachea and bronchi was carried out through a catheter during medium-depth inspiration under the X-ray control. For aerosol inhalations, we used a powder spraying device connected to a portable aerosol inhaler PAI-2 [5]. The number of insufflations required for the study ranged from 8 to

${ }^{*}$ Correspondence to: Ariel BM, St Petersburg Federal Research Institute of Phthisiology and Pulmonology, Healthcare Ministry of Russian Federation, Russia, E-mail: arielboris@rambler.ru

Key words: trachea, bronchi, exocrine glands, tantalum spraying, "risk zones", $X$-ray model

Received: October 08, 2018; Accepted: October 17, 2018; Published: October 19,2018 
15. We received the priority reference No. 2012147391 dated February 5, 2013 to the invention "Method of X-ray diagnostics of open cysts in the exocrine glands of the trachea and bronchi" [6]. Radiography of the lungs was performed immediately after spraying the particles in the trachea and bronchi as well as 24 and 48 hours after it. No complications associated with the procedure were noted.

\section{Results}

The investigation of the aerodynamics and peculiarities of inert sedimentation of tantalum microparticles colliding with the mucosa of the trachea and bronchi showed that at the bifurcations of the trachea and bronchi, where, according to the laws of aerodynamics, turbulent vortices on the surface of the mucosa are generated, the predominantly local particles precipitation in the form of multilayer bands up to $3 \mathrm{~mm}$ thick is observed. Identical deposition takes place on the lower walls of the main, lobar and segmental bronchi, as well as in the interspaces between folds that appear in the radiographs as areas of high optical density. Apparently, it is namely in these areas that the damaging effect of aerogenic particles on the tracheal and bronchial mucosa reaches its maximum, thus making it a "risk zone" liable to the development of a disease. Radiographs of 37 (54.4\%) patients taken immediately after spraying show the exact location of the "risk zones" in the trachea and bronchi, their dimensions, boundaries and the concentration of particles on the mucosal surface (Figures 1 and 2). The general scheme of "risk zones" incidence and location in the trachea and bronchi is given in Figure 3. We believe this picture represents the first stage of cyst formation in the wall of the trachea and bronchi.

It is easy to see that the multilayer accumulation of a huge quantity of different particles in the mucosal «risk zones» blocks the excretion process in protein and mucous glands and causes a diffuse dilation of their excretory ducts. Thus, X-ray images obtained in 19 (27.9\%) patients 24 and 48 hours after spraying revealed dilated excretory ducts of various shape (tapered, tubular, clavate and diverticula-like) in the "risk zones". These dilated ducts were localized at the border between cartilaginous and membranous parts of the wall and connected with the surface of the bronchial mucosa at an acute angle. This pattern seems to represent the second stage of cyst formation in the tracheal and bronchial walls.

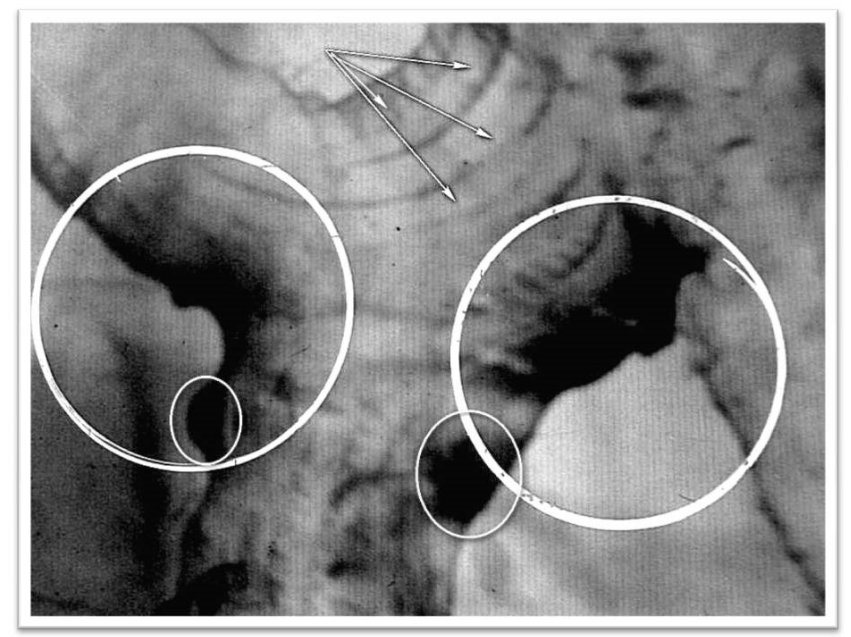

Figure 1. Bronchogram of the right lung (direct view) of patient M, 48 years old (a fragment). The microrelief of the mucous membrane and the "risk zone" on the bifurcation of the trachea, the lower walls of the right main, upper lobe and middle lobe bronchi (the area, boundaries, particle concentration)

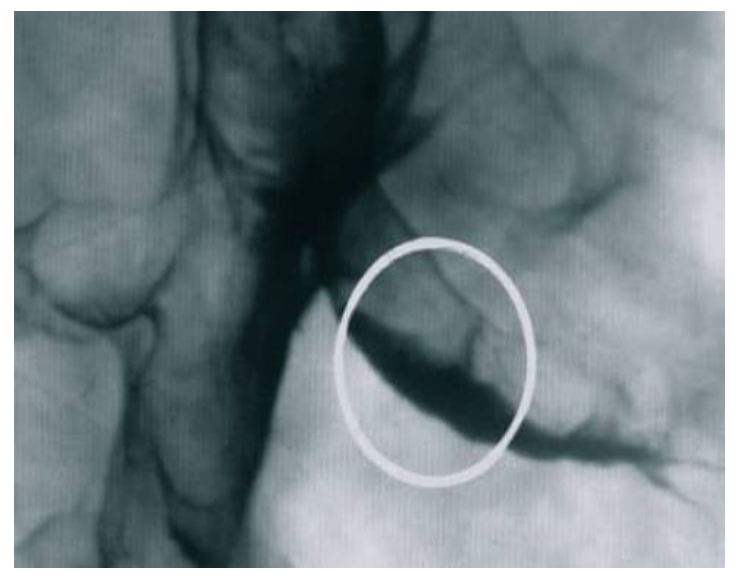

Figure 2. Bronchogram of the right lung (lateral view) of patient I, 37 years old (a fragment). Multilayer deposition of particles on the lower wall of the mid-lobar bronchus ("risk zone")

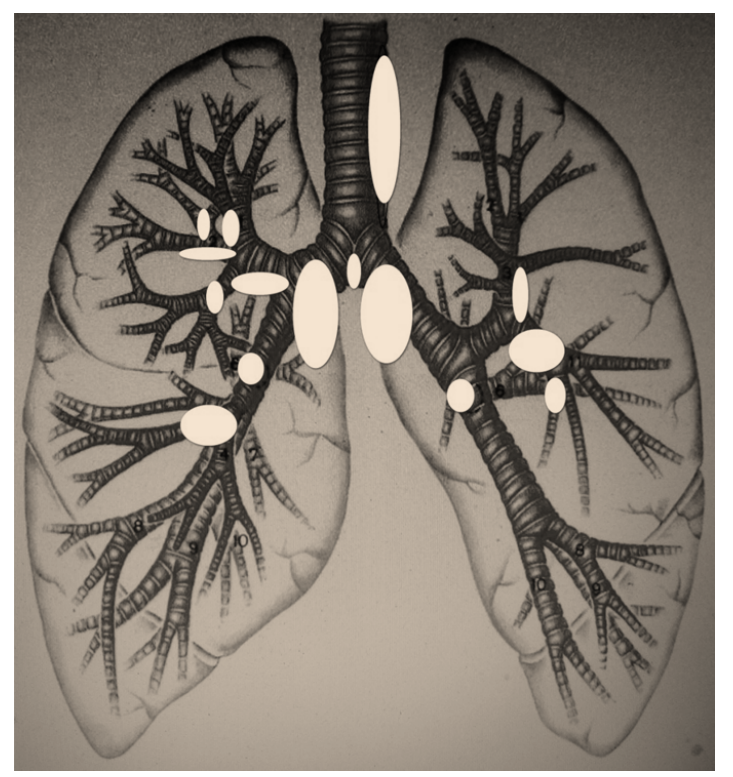

Figure 3. Scheme of location and incidence of "risk zones" on the mucosa of the trachea and bronchi

In addition to the above-mentioned dilated excretory ducts, open cysts of exocrine glands were found in the walls of the trachea and large bronchi of $12(17.1 \%)$ patients 24 and 48 hours after spraying (Figure 4). The cysts were localized in those areas where paratracheal, bifurcational and bronchopulmonary lymph nodes ajoined the walls of the bronchi. This picture corresponds to the third stage of cysts formation in the wall of the trachea and bronchi.

Cysts were filled with tantalum particles by way of diffusion of the latter through dilated excretory ducts and their sedimentation on the walls by virtue of Brownian movement. The cysts had round or oval shape; they appeared in the trachea (1 cyst) as well as in the main bronchus (6), the intermediate (2) and the lobar (3) ones. Their dimensions ranged from 3 to $10 \mathrm{~mm}$ in size and from 1 to 6 in number (Figures 5 and 6). Cysts were arranged longitudinally at the border between the membranous and the cartilaginous parts.

Presumably, open cysts are derived from closed ones when their contents are removed as a result of reflex contraction of the wall muscles that squeeze secret out into the lumen of the trachea and bronchi. In this case, unlike the diverticula located in the walls of the trachea and 


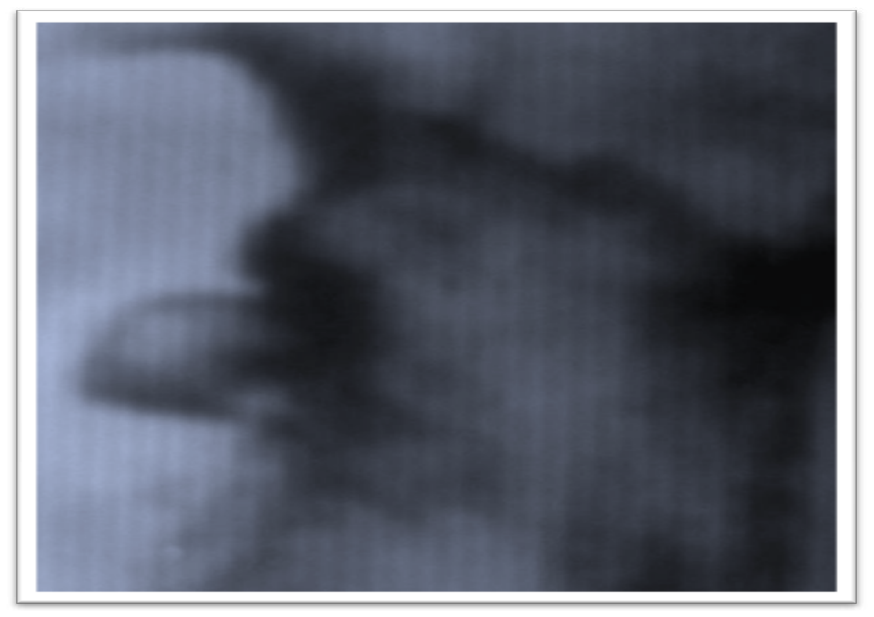

Figure 4. Bronchogram of the left lung (direct view) of patient $S, 35$ years old (a fragment). In the risk zone" of the lower wall of the lingual bronchus there are 3 cysts filled with tantalum particles $4 \mathrm{~mm}, 8 \mathrm{~mm}$ and $5 \mathrm{~mm}$ in size; a narrow excretory duct

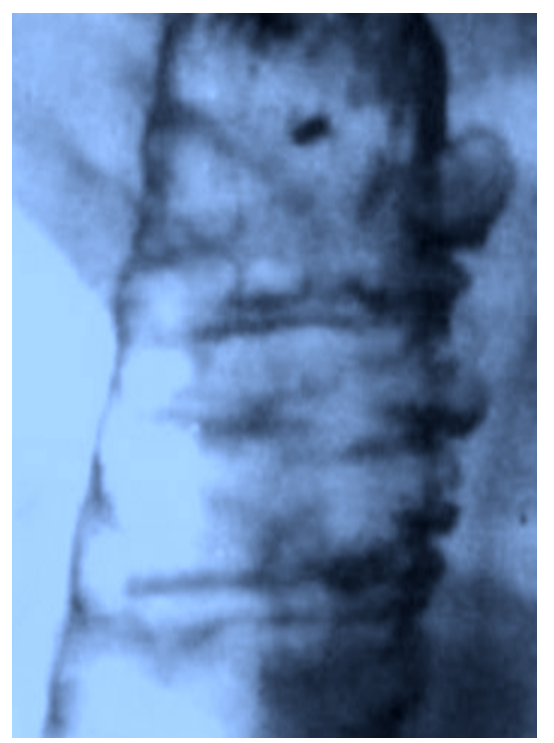

Figure 5. Bronchogram of the right lung (direct view) of patient L, 49 years old (a fragment). A cyst in the "risk zone" of the intermediate bronchus with a diameter of $8 \mathrm{~mm}$; a wide excretory duct

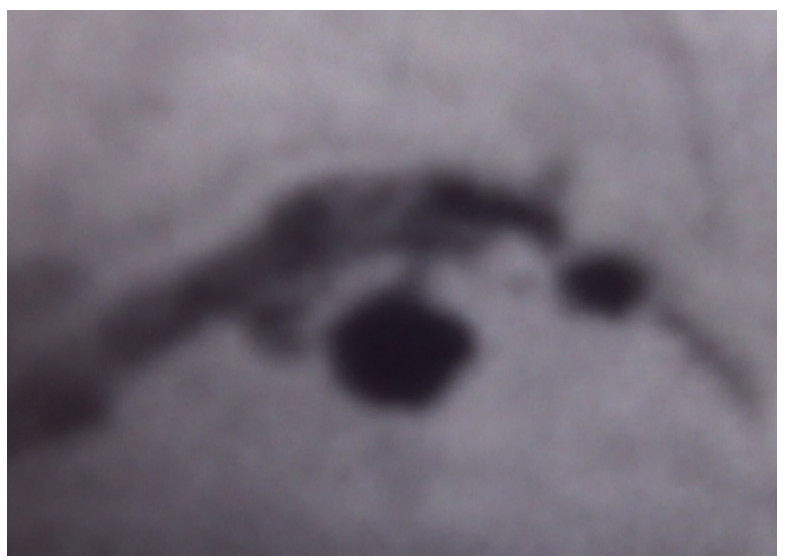

Figure 6. Tracheogram (direct view) of the patient R, 53 years old (a fragment). Polycystosis in the "risk zone" on the left side of the trachea at the border between the cartilaginous and the membranous parts. Dimensions of the cysts vary from 3 to $10 \mathrm{~mm}$ bronchi, the size of the ducts and cysts shape vary, which is clearly defined on the radiographs taken 24 and 48 hours after spraying.

\section{Conclusion}

1. There are "risk zones" in the walls of the trachea and major bronchi where the cysts of the exocrine glands develop. They serve as a depot in which the contents of the respiratory tract (including toxic particles, carcinogens, infectious agents, and the like) are retained for a long time and from whence the contents of the bronchi get into other parts of the respiratory tract in the shortest possible way.

2. Cysts of glands located in the walls of the trachea and major bronchi serve as an anatomical base for the development of tumors, infectious diseases and other pathologies.

3. Precipitation of tantalum microparticles in the "risk zones" and in the cysts located in the wall of the trachea and large bronchi provides a standard X-ray model for studying the physiological mechanisms of the interaction between aerogenic microparticles of various nature and the mucous membrane of the respiratory tract.

\section{Acknowledgement}

We acknowledge Alexei Mikhailov, Department of Pathology, Wake Forest University, Winston-Salem, NC, USA

for his expert help with manuscript preparation.

\section{References}

1. Spencer's Pathology of the Lung. 5th ed (1996) H.S.Hasleton (ed.) McGraw-Hill. 1283

2. Sapin MR, Nikitiuk VB, Shadlinsky DB, Movsumov TN (2001) Small glands of the digestive and respiratory systems Elista: APP "Dzhangar" 136.

3. Kriukov AI, Kirasirova EA, Romanenko SG, Eliseev OV (2008) Experimental model for the evaluation of the method and regimen of inhalation therapy. Vestn Otorinolaringol 42-44. [Crossref]

4. Fedorovich GV (2013) The role of the inertial mechanism in the process of air purification in the lungs from aerosol particles. Pulmonology 2: 114-118.

5. Krishtafovich AA, Rabinkin VD, Zhukov S (1996) The device for inhalation of powdery drugs. J Publ. Bul. No. 4, Pat. RF № 2053801. IPC AND 61 IN 6/00.

6. Krishtafovich AA, Kryvokhizh VN (2013) A method of X-ray diagnosis of open cysts of exocrine glands of the trachea and bronchi. Spb State Pediatric Medical Academy, Ministry of health and social development of Russia. Priority reference №2012147391.

Copyright: (C2018 Krishtafovich AA. This is an open-access article distributed under the terms of the Creative Commons Attribution License, which permits unrestricted use, distribution, and reproduction in any medium, provided the original author and source are credited. 